\title{
Research on Biological Snapshot Technology Based on Telemedicine Architecture
}

\author{
Mayilamu Musideke ${ }^{1, a}$, Shentong $\mathrm{Mo}^{2, \mathrm{~b}}$, Qiang Zou ${ }^{2, \mathrm{c}}$, \\ Dong $\mathrm{Jia}^{1, \mathrm{a}}$ and Luanyu Song ${ }^{3, \mathrm{a}}$
}

${ }^{1}$ Xinjiang Uygur Autonomous Region Fiber Inspection Bureau, New Urban District, Urumqi, 830011, P. R. China

${ }^{2}$ School of electronic and information engineering, Tianjin University, Nankai District, Tianjin, 300072, P. R. China

${ }^{3}$ Xinjiang Infotool CO.,LTD, 2nd Science Street, Urumqi, 830011, P. R. China

amahiralaser@163.com, bmoshentong678@126.com, 'couqiang@tju.edu.cn

\begin{abstract}
Keywords: Cancer; Raman spectroscopy; UV spectrophotometry; Telemedicine; Biological Snapshot

Abstract. Cancer has always been possessing the highest mortality rate among major diseases, therefore, it is necessary and revolutionary for the research of the prevention of cancer. World Health Organization tells us that at least $30 \%$ of cancer is preventable and can be found in advance, so how to detect the cancer rapidly and sensitively has far-reaching significance. In this article, we used the UV spectrophotometric detection technology for human IgG protein and Raman spectroscopy to do a large number of experimental studies for qualitative and quantitative protein, obtained Raman spectra of characteristic protein and dyestuff, as well as UV spectrophotometer measured spectrum peak. We analyzed the experiment the get the conclusion, and established the database of the Raman spectra of proteins, so as to work in with telemedicine to improve current method of cancer's detection and diagnosis. Only with a complete test system program, will the rest of telemedicine have accurate and reliable data to support further depth promotion.
\end{abstract}

\section{Introduction}

Surgical treatment of tumors of the concept enjoys popular support, however, the World Health Organization in 1984 declared one-third of cancers which can be prevented. One-third of cancer by early detection can be found, earlier diagnosis and early treatment can led to recovery; one-third of cancer can alleviate the suffering of patients through effective treatment and prolong its life [1-3]. Ministry of Health in 1996 has also developed a "prevention first, combining prevention with control, focusing on" three early "(early detection, early diagnosis, early treatment) strategic approach to achieve cancer control [4-5]. Cancer is a common chronic disease mortality which has been ranking first in all kinds of causes of death, cancer is already present endanger people's lives first major diseases, while the world's annual new tumors occurred in $80 \%$ of China, India, Brazil and other developing countries, in the course of treatment after long and found a patient in need enormous financial and energy, which accentuated the importance of cancer prevention.

Current Chinese method of early diagnosis of cancer is followed by B, X-ray examination, endoscopy and blood. B Ultra can detect kidney, liver, pelvic tumors such as endometrial cancer, pelvic cancer, ovarian cancer and the like. While X-ray can be screened for lung cancer [6-8]. Endoscopy for checking colon cancer, stomach cancer. Blood is suitable for the prostate screening. B-clarity, resolution, etc. weaker than $\mathrm{CT}$, and other hollow organs and intestinal diseases easily missed, pregnant women may be prone to cause indiscriminate check B ultrasound fetal malformation. X-ray examination: the need to multi-position shooting function is not directly observed, cumbersome procedures, high costs, endoscopy and blood: the process will be painful discomfort [9-10]. 
Functional genomics strategies have been applied to the detection of tumor biomarkers, including gene expression and protein profiling. In recent years, an increasing number of metabolomics research shows that bodily fluid metabolites also may serve as a marker for early detection or monitoring of a variety of tumor progression, such as breast cancer, colon cancer, liver cancer, stomach cancer, prostate cancer [11]. Over the years, the early discovery and early diagnosis of malignant tumors has been a lot of research and found a number of serum tumor markers, and as an important means of diagnosis of cancer, but the presence of serum tumor markers complicated operation, cost high, invasive, early detection rate is not ideal and other factors, is not conducive to tumor mass screening, therefore, found that a drawn convenient, easy operation, non-invasive, and can be used for a broad spectrum of early cancer screening the detection method is very important [12-13]. Easy to collect urine samples and non-invasive, patient compliance is high, so many medical experts and scholars dedicated to the research of cancer urine test, the current detection of the urine is divided into two: one is the detection of dopa, other One is the detection of monohydric phenol derivative.

Japanese researchers recently developed a chip to check for cancer, with a drop of urine can detect early cancer, the future is expected to be used as a simple inspection equipment clinics and family. According to the "Japan Industrial News" reported that, after people suffering from cancer, there is some urine is called "cancer marker" protein, thus early detection of cancer can be found by urine tests. However, due to the concentration of urine change easily, and therefore also need to check the content of the urine can show concentrations of creatine, the entire testing process is too much trouble [14]. Japan's Industrial Technology Research Institute research team developed a new chip, with a drop of urine can also check the content of the "cancer markers" of creatine and 5 minutes to know the results. Chip assembly has two sensors, respectively, for detecting "cancer markers" and creatine. The researchers expect that after two or three years can be practical means.

Researchers at the University of Edinburgh has recently adopted a new method for detecting levels of certain proteins in urine to diagnose upper gastrointestinal cancer development, we can more accurately diagnose bowel cancer, stomach cancer and pancreatic cancer, and other related cancers [15]. This discovery will help cancer patients for early diagnosis and prompt treatment. Their digestive tract cancer patients and 60 healthy people on 60 urine were compared and found that some of the protein content of the obvious differences. Wherein code-named S100A6 and S1009 indicative of six protein is particularly evident in the urine of $98 \%$ of cancer patients can find their tracks, while more than $90 \%$ of healthy people do not have these proteins in urine.

\section{Experimental Methods}

UV-spectrophotometric determination of protein content. Turn on the printer, start the computer, turn on the mains switch (in color plate light). UV double-click the icon to start the workstation and initialize the instrument. Determination of project work interface selection (spectral scanning, photometric measurements), this experiment selected photometric measurements, set measurement conditions (measurement wavelength, etc.), the instrument to warm up 30min. Blank into the test chamber, click the Start scan blank and click ZERO. The sample is placed in the measurement unit, click Start scanning spectrum, click the READ absorbance measurements. Quantitative determination to set a standard sample (sample number, concentration fitting size, etc.). Data save (or print the results). Leave the machine, shut down the host, shut down the computer (and printers).

Human IgG protein solution pipette amount of $2 \mathrm{~mL}$ of $5.00 \mathrm{mg} / \mathrm{mL}$ to $10 \mathrm{ml}$ colorimetric tube, and $0.9 \% \mathrm{NaCl}$ solution was diluted to the mark, shake. Using $1 \mathrm{~cm}$ quartz plate, with a $0.9 \% \mathrm{NaCl}$ solution as a reference, in the $190 \mathrm{~nm} \sim 400 \mathrm{~nm}$ spectral band scan interval is measured, the absorption rate.

Mobile $5.00 \mathrm{mg} / \mathrm{mL}$ human IgG protein solution 50,100,150,200 and $250 \mathrm{ml}, 500 \mathrm{ml}$ cuvette with $0.9 \% \mathrm{NaCl}$ solution was diluted to the mark, shake. A standard solution is to determine the absorbance of the standard solution as reference points, and measuring the absorbance at a maximum absorption wavelength of $278 \mathrm{~nm}$ solution standard solution in $0.9 \% \mathrm{NaCl}$ solution. 
Accurately shifted to the protein solution in $3 \mathrm{ml} 10 \mathrm{ml}$ colorimetric test tube, the solution was diluted with $0.9 \% \mathrm{NaCl}$ to the mark, jitter. The absorbance at the maximum absorption wavelength is determined by the above method. Parallel measured three times.

Raman Spectroscopy. According to previous experiments have to dye it the best experimental substrate, and then using various concentrations of protein were detected here is worth noting that, because of the large fluorescent protein solution, so we need to give the protein solution is diluted, general the case of the human body is a normal protein content, so $1.5 \mathrm{mg} / \mathrm{ml}$ is a high concentration of the protein solution, so we measured a group of $1.5 \mathrm{mg} / \mathrm{ml}$, the protein solution is then detected $0.75 \mathrm{mg}$ $/ \mathrm{ml}$, and then find a low concentration time better, because that will increase the fluorescent protein, will increase the reflection of incident light and the Raman effect of refraction, which is required to accept the light intensity Raman detection is not good news, so we can be within the measuring range minimize protein concentration is best.

\section{Results and Discussion}

Containing conjugated double bonds, the protein has the ability to absorb ultraviolet protein molecule tyrosine, phenylalanine and tryptophan residues of benzene. Absorption peak at 280nm wavelength. All proteins containing tyrosine, so the absorption at $280 \mathrm{~nm}$ is a common characteristic of the protein. To a certain extent, the absorbance at $280 \mathrm{~nm}$ protein solution is directly proportional to its concentration, it can be used for quantitative determination of proteins. The most commonly used UV absorption is $280 \mathrm{~nm}$. Protein solution containing a nucleic acid is to absorb the difference in the method at $280 \mathrm{~nm}$ and $260 \mathrm{~nm}$ better.

Table 1 protein titration experiments linear programming curve data

\begin{tabular}{ccccccc}
\hline No. & Serial number Types & Concentration & Abs & SD & RSD \\
\hline 1 & $1.25 \mathrm{mg} / \mathrm{ml}$ standard sample & 1250.0000 & 0.049 & 0.049 & 0.0000 & 0.0000 \\
2 & $1.00 \mathrm{mg} / \mathrm{ml}$ standard sample & 1000.0000 & 0.038 & 0.038 & 0.0000 & 0.0000 \\
3 & $0.75 \mathrm{mg} / \mathrm{ml}$ standard sample & 750.0000 & 0.031 & 0.031 & 0.0000 & 0.0000 \\
4 & $0.50 \mathrm{mg} / \mathrm{ml}$ standard sample & 500.0000 & 0.01 & 0.017 & 0.0000 & 0.0000 \\
5 & $0.25 \mathrm{mg} / \mathrm{ml}$ standard sample & 250.0000 & 0.006 & 0.006 & 0.0000 & 0.0000 \\
\hline
\end{tabular}

By the wavelength of $215-225 \mathrm{~nm}$ and a differential absorption method of dilute protein solution, the protein concentration in the range of per unit volume of $20-100 \mathrm{ug} / \mathrm{L}$, and the absorbance is proportional to the protein concentration, hydroxide $0.1 \mathrm{~mol} / \mathrm{L}$ sodium acetate, succinic acid, phthalic dimethyl acids, barbiturates, such as buffer $215 \mathrm{~nm}$ absorbance the greater the concentration must be decreased to $0.005 \mathrm{ug} / \mathrm{L}$ no significant response. The method of determining the peptide bond is larger than 280nm, which is sensitive to light absorption, but a variety of organic compounds such as alcohols, aldehydes, ketones, ethers, organic acids, amides and hydrogen peroxide interference, so it is best to use an inorganic salt, an inorganic base and water was measured. UV absorption method is simple, sensitive, rapid, do not consume the sample, a low concentration of salt does not interfere with the determination, the disadvantage is the standard protein tyrosine and tryptophan content of proteins are quite different, there are certain errors. Said nucleic acid having a strong absorption in the ultraviolet region, but can be eliminated by calibration. 


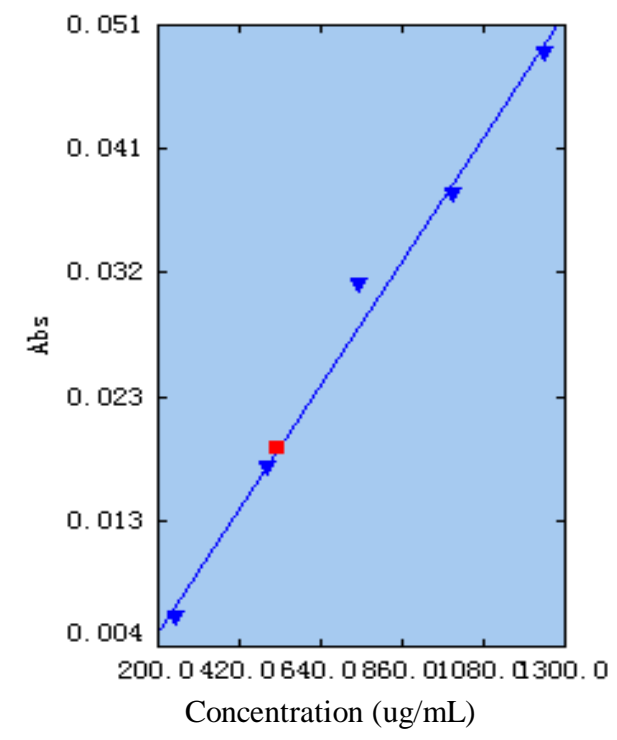

Fig.1 Linearity curve between absorbance and protein concentration

As can be seen in Fig.1, the most suitable measurement wavelength absorption curve is $278 \mathrm{~nm}$. Absorbance for the vertical axis, the horizontal coordinate is the protein concentration to a standard curve. By Origin and plotted to obtain the ultraviolet spectral image of the human IgG protein, protein because it is the reason, and it is a very good response by UV peak is low, wave peak signal to noise ratio is high. As can be seen from Fig.2, a high signal to noise ratio, peaks are obtained by UV the human IgG protein characteristic peaks.

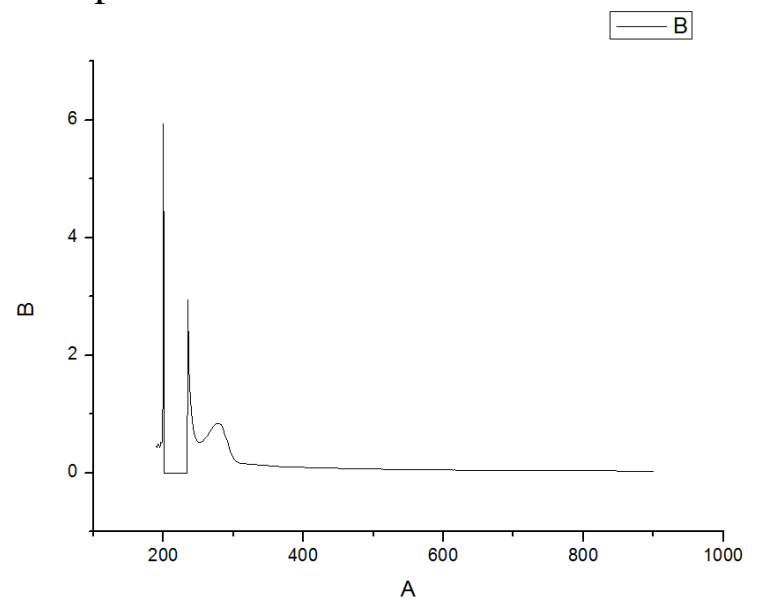

Fig.2 UV spectral image of the human IgG protein

By UV spectrophotometric determination of protein content, the advantage of simple, sensitive, rapid detection and high selectivity, and good stability, no sample consumption, low concentrations of different salts of interference measurement. The disadvantage is that expensive equipment, and different proteins UV absorption is not the same, there are some errors, and the light, the effect of the limited color solution cell substances, buffers, and other factors $\mathrm{pH}$. 

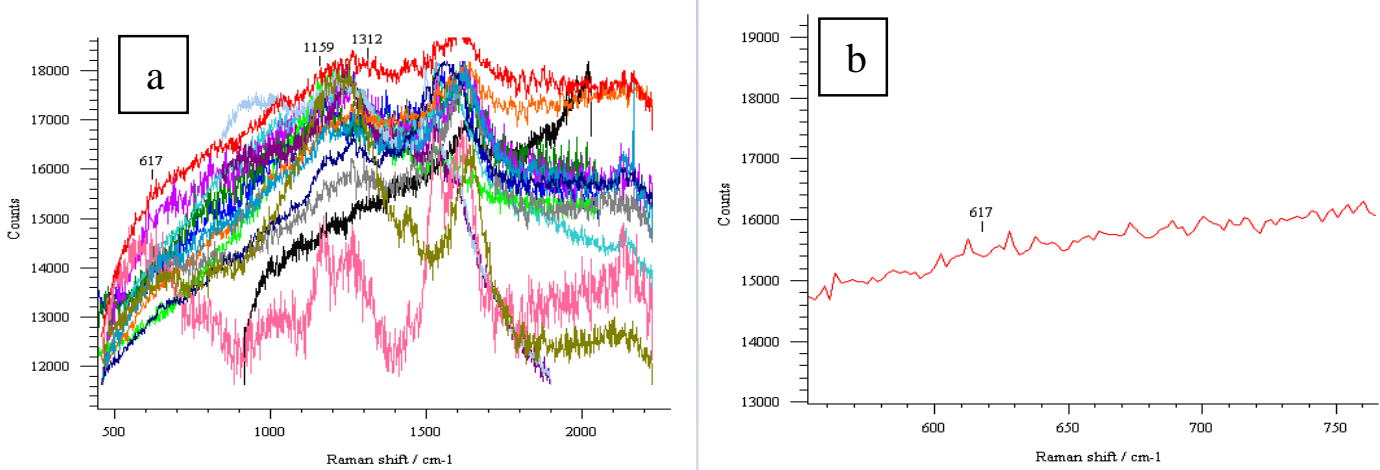

Fig.3 (a) Raman spectra of different substrates; (b) the best peak Raman spectra of human $\mathrm{IgG}$ protein

As can be seen from Fig.3, the Raman feature of human IgG protein peaks at about 617, and after a lot of testing that can be measured, it is very remarkable breakthrough,

\section{Conclusions}

It is significantly easier to do the Raman detection of dyestuff than the protein. The reason is that a dye solution belongs to a solution, its particle size is nanometer-level. The protein solution belongs to colloid, which has greater impact on reflection and refraction, so it can absorb more light. But we found that the smaller the concentration of the protein solution, the better the test results of Raman spectra. The theorem is to weaken its fluorescent properties. In addition, as the protein is biologically active molecules, which is detected when the water can not be used. This will lead to inactivation of proteins, which can affect the accuracy of test results. But sodium chloride may affect the results. This is where the difficulty lies.

However, what excited us is that, when the protein solution was almost dry, we pleased to find the protein crystallization and sodium chloride crystals were separated under electron microscopy. Sodium chloride crystallization is green, while protein is blue with a small amount of aggregation, which is a dipeptide or polypeptide molecules. Use Raman to detect the position of the blue, when the effect is a lot better than the effect of direct measurement of the solution, which shows clear peaks. The reason is that light can penetrate completely illuminated peaks such reference value of protein is relatively large.

The experimental design of complete protein, dye calibration and qualitative detection of the concentration of the substance to distinguish the type of material and proposes a fast reliable research programs, following Raman spectroscopy can continue to explore other kinds of protein FIG and ultraviolet spectral diagram of the telemedicine program provides a large part of the data accumulated reliable data. Rapid screening program design, the use of large amounts of data make up the database, and complete the data faster than the optimal solution to achieve rapid detection of biological information, provide some technical support for the rapid detection of biological technology.

\section{Acknowledgements}

This work was financially supported by National Science and Technology Support Project (2015) and the collaborative innovation projects in Nankai District (2014).

\section{References}

[1] Vadivel Masilamani, Trinka Vijmasi, Mohammad Al Salhi et al. Journal of Biomedical Optics, 15(5):1-9 (2010).

[2] Jun P, Chen Y T, Chen C L et al. Anal. Chem., 86, 6540-6547(2014).

[3] Luis P. Fonseca, Verónica C.B. Martins, Paulo P. Freitas. In: 1st Portuguese Meeting in Bioengineering. Lisbon, Portugal:1-4 (2011). 
[4] Ahmed IbrahimNossier, Sanaa Eissa, Manal FouadIsmail et al. Biosensors and Bioelectronics, 54: 7-14 (2014).

[5] Liliana A.A.N.A. Truta, Nádia S. Ferreira, M. Goreti F et al. Electrochimica Acta, 150, 99-107 (2014).

[6] Tessy Theres Baby, Sundara Ramaprabhu. In: International Conference on Nanoscience, Technology and Societal Implications. Bhubaneswar, India:1-6 (2011).

[7] P. Palestri, R. Sette, F. Pittino. In: 14th International Conference on Ultimate Integration on Silicon. Warwick, United Kingdom:77-80(2013).

[8] Kiyoshi Takamatsu, Satoru Kaneko, Keisuke Miyaji et al. J. Sep. Sci., 30, 2279-2285 (2007).

[9] Liu Q, Shin Q, Jack Sheng Kee et al. Biosensors and Bioelectronics, 71:365-372 (2015).

[10] David Maraldo, Fernando U. Garcia, Raj Mutharasan. Anal. Chem. 79:7683-7690 (2007).

[11] T.R.M. De Beer, P. Vercruysse, A. Burggraeve et al. Journal of Pharmaceutical Sciences, 98 (9), 3430-3446 (2009).

[12] Hamid Hashemi-Moghaddam, Majid Rahimian, Bahman Niromand. Bull. Korean Chem. Soc., 34(8):2330-2334 (2013).

[13] Sitansu Sekhar Nanda, Min Jik Kim, Kwi Seok Yeom et al. Trends in Analytical Chemistry, 80, 125-131 (2016).

[14] Huang L L, Guo L, Wan Y Qet al. Molecular and Biomolecular Spectroscopy, 120: 595-601 (2014).

[15] Cristina Sottani, Massimo Zucchetti, Marco Zaffaroni et al. Rapid Commun. Mass Spectrom, 18: 1017-1023 (2004). 